\section{DNA break-induced sumoylation is enabled by collaboration between a SUMO ligase and the ssDNA- binding complex RPA}

\author{
Inn Chung and Xiaolan Zhao \\ Molecular Biology Program, Memorial Sloan Kettering Cancer \\ Center, New York, New York 10065, USA
}

Upon genome damage, large-scale protein sumoylation occurs from yeast to humans to promote DNA repair. Currently, the underlying mechanism is largely unknown. Here we show that, upon DNA break induction, the budding yeast SUMO ligase Siz2 collaborates with the ssDNA-binding complex RPA (replication protein A) to induce the sumoylation of recombination factors and confer damage resistance. Both RPA and nuclease-generated ssDNA promote Siz2-mediated sumoylation. Mechanistically, the conserved Siz2 interaction with RPA enables Siz2 localization to damage sites. These findings provide a molecular basis for recruiting SUMO ligases to the vicinity of their substrates to induce sumoylation upon DNA damage.

Supplemental material is available for this article.

Received May 11, 2015; revised version accepted July 9, 2015.

Increasing genome protection in response to clastogen exposure is a conserved function of the cell and relies on waves of post-translational modifications (PTMs) that change properties of many proteins. These modifications coordinate multiple pathways in a process collectively known as the DNA damage response (DDR). The beststudied PTM responses are mediated by the DNA damage checkpoint kinases, which accumulate at DNA lesion sites, activate signaling cascades, and phosphorylate hundreds of substrates. Recent studies have revealed critical roles for other types of PTMs in DDR. In particular, protein sumoylation promotes DNA repair and other PTM responses in both yeast and humans. In human cells, sumoylation enzymes arrive at DNA lesion sites shortly after treatment with double-strand break (DSB)-inducing agents and are required for DSB repair by homologous recombination (Galanty et al. 2009; Morris et al. 2009). In yeast, sumoylation promotes several steps in DSB repair and targets multiple recombination proteins (for review, see Sarangi and Zhao 2015). In both organisms, the conserved PIAS family SUMO ligases are required for DSB repair situations (Galanty et al. 2009; Psakhye and Jentsch 2012). How these SUMO ligases are recruited to DSB sites

[Keywords: homologous recombination; DNA damage response; protein sumoylation; DNA break resection]

Corresponding author: zhaox1@mskcc.org

Article is online at http://www.genesdev.org/cgi/doi/10.1101/gad.265058. 115. and induce sumoylation of repair proteins is not well understood.

Each of the PIAS family SUMO ligases, such as the budding yeast Siz1 and Siz2 and human PIAS1-4 proteins, contains an N-terminal SAP (SAF-A/B, Acinus, and PIAS) domain, which is generally known for supporting DNA or protein interactions (for review, see Rytinki et al. 2009). The SAP domain of the PIAS family is uniquely composed of a core SAP (cSAP) region shared with other SAP-containing proteins and a one- to two-a-helix extension (eSAP) found only in this family of proteins (Okubo et al. 2004; Suzuki et al. 2009). SAP deletion abolishes human PIAS accumulation at DSB sites, indicating its importance in targeting the enzymes to lesion sites /Galanty et al. 2009). Further studies in yeast suggest that, for the Siz2 protein, both DNA binding via its CSAP and a C-terminal SUMO-interacting motif (SIM) are important for its function when cells are treated with the DNA methylation agent MMS (methyl methanesulfonate) (Psakhye and Jentsch 2012). Whether these are generally required for Siz2/PIAS-mediated sumoylation upon DNA damage (for example, in DSB situations) is not known. Illuminating this issue will be important to understand an early and essential step of sumoylation induction during DDR.

Here we examine how the Siz2 SUMO ligase is recruited to DNA lesions and elicits the sumoylation of recombination proteins in response to the radiomimetic agent zeocin that generates DSBs. We show that the previously characterized DNA-binding residues in the cSAP region and the C-terminal SIM of Siz2 are not required for zeocin-induced sumoylation. Instead, we uncovered a novel and conserved interaction between Siz2 and the ssDNAbinding complex RPA (replication protein A), a wellknown sensor of DNA lesions that recruits checkpoint kinases and ubiquitin enzymes. Genetically, both RPA and ssDNA generation through resection are required for DSB-induced sumoylation, indicating the importance of RPA-coated ssDNA for this process. We determined that key residues in Siz2 for RPA interaction lie mainly in the eSAP domain and are required for damage-induced sumoylation and damage resistance. Mechanistically, the RPA-Siz2 interaction enables Siz2 recruitment to DNA damage foci and chromatin. These findings suggest that the initial recruitment of SUMO ligases to DNA lesion sites is achieved by a specific interaction between these enzymes and RPA that coats ssDNA and that cells use a unified paradigm to coordinately recruit multiple PTM enzymes during DDR.

\section{Results and Discussion}

\section{Siz2 sumoylates recombination proteins in response to zeocin}

To investigate the mechanism of DNA break-induced sumoylation, we chose the SUMO ligase Siz2 as a model enzyme and examined cells after treatment with zeocin

(C) 2015 Chung and Zhao This article is distributed exclusively by Cold Spring Harbor Laboratory Press for the first six months after the full-issue publication date (see http://genesdev.cshlp.org/site/misc/terms.xhtml). After six months, it is available under a Creative Commons License (Attribution-NonCommercial 4.0 International), as described at http:// creativecommons.org/licenses/by-nc/4.0/. 
that primarily generates DSBs. It is known that Siz2 can sumoylate the recombination mediator Rad52; its binding partner, Rad59; and the large subunit of the heterotrimer RPA, Rfa1, in response to MMS treatment (Psakhye and Jentsch 2012). We found that, in response to zeocin, Siz2 loss strongly reduced the appearance of monosumoylated and disumoylated forms of Rfa1 and Rad52 and disumoylated Rad59, while the loss of the homologous Siz1 had no effect (Fig. 1A,B). As mutating two critical residues in the SUMO ligase domain (or SP-RING) in the siz2-CH mutant (C354/H356A) (Johnson and Gupta 2001) had the same effects as siz2d (Fig. 1C; Supplemental Fig. $\mathrm{S} 1 \mathrm{~A})$, the ligase activity of Siz2 is responsible for these sumoylation events. We note that the monosumoylated form of Rad59 and residual Rfa1 and Rad52 sumoylation seen in siz2 $\Delta$ and siz2- $\mathrm{CH}$ mutants likely reflect the contribution of additional SUMO ligases.

Siz2-mediated sumoylation does not rely on its cSAP DNA interaction or C-terminal SIM

Next, we tested how Siz2 cSAP-mediated DNA binding and its C-terminal SIM affected zeocin-induced sumoylation. Mutating three conserved residues in the cSAP domain of Siz2 (cSAPmut: G64/K66/L69A) has been reported to disrupt DNA interaction in vitro and reduce Siz2 protein levels in vivo (Psakhye and Jentsch 2012). We confirmed both findings (Supplemental Fig. S1B,C). Importantly, after adjusting the protein level to that of wild type using a galactose-inducible promoter, sumoylation of Rfa1, Rad52, and Rad59 in siz2-cSAPmut cells after zeocin treatment was similar to that in wild-type cells (Fig. 1D; Supplemental Fig. S1D). In addition, siz2-cSAPmut did not affect Siz2 autosumoylation (Supplemental Fig. S1D-F). Thus, the DNA-binding function of cSAP is not critical for Siz2-mediated sumoylation of both itself and its substrates in response to DSBs. Moreover, the Siz2 C-terminal SIM mutation characterized previously (V720/V721A) (Fig. 1C; Psakhye and Jentsch 2012) also sustained the sumoylation of the tested substrates and Siz2 itself upon zeocin treatment (Fig. 1E; Supplemental Fig. S1G), suggesting that the C-terminal SIM is dispensable for DNA break-induced sumoylation.

\section{Siz2 interacts with the middle subunit of the RPA complex}

To gain insight into Siz2-mediated sumoylation, we tested interactions between Siz2 and its substrates and the obligatory binding partners of Rfa1 (namely, Rfa2 and $\mathrm{Rfa} 3$ ) in a yeast two-hybrid ( $\mathrm{Y} 2 \mathrm{H})$ assay. We detected an interaction of Siz2 only with Rfa2 (Fig. 2A). All constructs used were functional, as they supported known interactions (Supplemental Fig. S2A; data not shown). We further narrowed down the interacting region of $\mathrm{Rfa} 2$ to its $\mathrm{C}$-terminal region containing a winged helix $(\mathrm{WH})$ domain (Supplemental Fig. S2A). Moreover, the observed Rfa2 interaction was specific to Siz2, as no interaction was seen with Siz1 (Fig. 2A), which did not affect the sumoylation of the tested proteins (Fig. 1A).

We confirmed the observed $\mathrm{Y} 2 \mathrm{H}$ interaction by coimmunoprecipitation (co-IP) using endogenously expressed proteins. As the three RPA subunits form a stable complex in cells, the reproducible enrichment of Rfal in the Siz2 immunoprecipitated fraction suggests that Siz2 associates
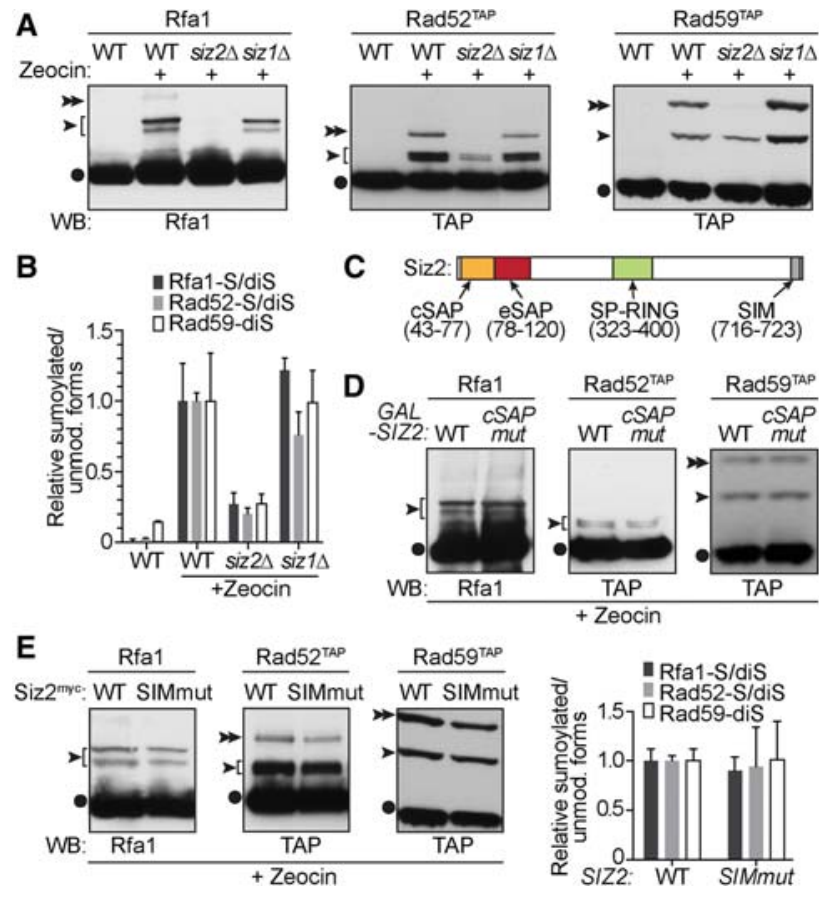

Figure 1. The Siz2 SUMO ligase enables specific sumoylation in response to zeocin treatment. $(A, B)$ Zeocin-induced sumoylation of Rfa1, Rad52, and Rad59 requires Siz2. (A) Cell lysates were examined by Western blotting using antibodies against the protein or the tag. Unmodified proteins are labeled with dots, and monosumoylated and disumoylated bands are labeled with single and double arrowheads, respectively. $(B)$ Quantifications of Western blots from three trials with mean and SD. The graph indicates the ratio of sumoylated ([-S] monosumoylated; [di-S] disumoylated) to unmodified bands normalized to wild type (WT) treated with zeocin. $(C)$ Schematic of Siz2 domains and amino acid coordinates (for details, see the text). (D) siz2-cSAPmut sustains sumoylation of substrates after zeocin treatment. Galactose-inducible alleles of SIZ2 and siz2-cSAPmut were used to obtain similar protein levels, as shown in Supplemental Figure S1D. (E) Mutation of the C-terminal SIM of Siz2 (SIMmut) does not affect zeocin-induced sumoylation of substrates. Quantification was done as in $B$.

with the RPA complex in cells (Fig. 2B). The interaction was insensitive to nuclease treatment and moderately enhanced upon zeocin treatment (Fig. 2B). Considering that RPA enables DNA lesion sensing and Siz2 interacts only with RPA but not Rad52 or Rad59, we hypothesized that the Siz2-RPA interaction may not only be responsible for RPA sumoylation but also plays a broader role by recruiting the Siz2 ligase to promote sumoylation of its other substrates, such as Rad52 and Rad59.

\section{$R P A$ is required for Siz2-mediated sumoylation of Rad52 and Rad59}

One important prediction of the above hypothesis is that, besides Siz2, RPA should also be required for Rad52 and Rad59 sumoylation. To test this, we used an Rfal degron (Rfa1-AID) that acutely depleted the protein in the presence of both indole-3-acetic acid (IAA) and the cofactor TIR (Supplemental Fig. S2B; Nishimura et al. 2009). Note that the lack of any one subunit of RPA abolishes the function of the complex (Brill and Stillman 1991). Upon RPA depletion, zeocin-induced sumoylation of 


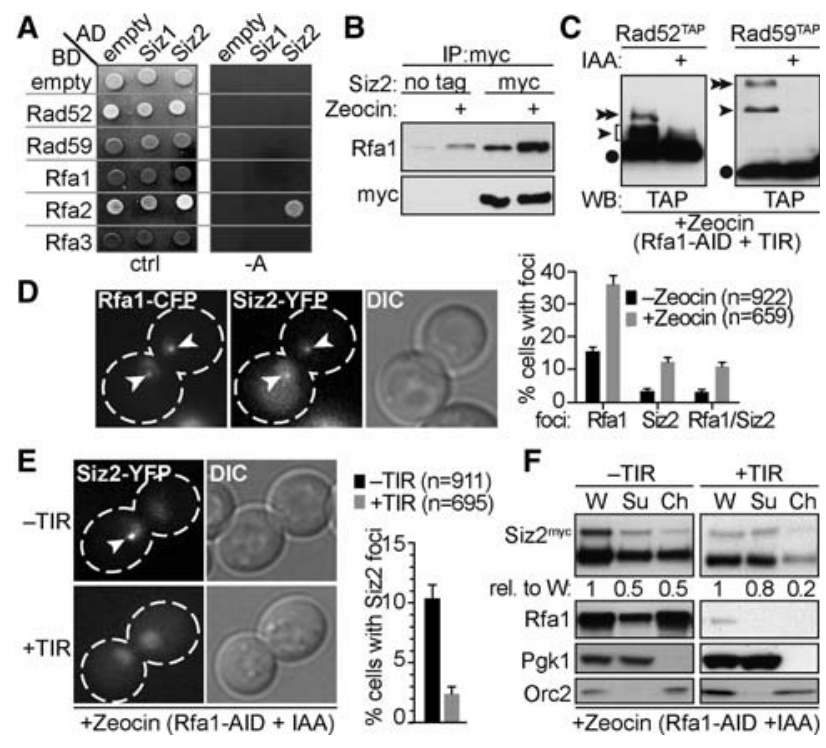

Figure 2. Siz2 interacts with RPA, and RPA depletion results in loss of Siz2-mediated sumoylation, Siz2 foci, and chromatin association. (A) Rfa2 interacts with Siz2 in $\mathrm{Y} 2 \mathrm{H}$ assays. All constructs support growth on control (ctrl) plates, but only the ones that interact support growth on -A plates. (B) Siz2 interacts with RPA in coimmunoprecipitation assays. Myc-tagged Siz2 was immunoprecipitated, and RPA was detected with an anti-Rfal antibody. (C) RPA depletion abolishes zeocin-induced Rad52 and Rad59 sumoylation. Cells were incubated with $1 \mathrm{mM}$ indole-3-acetic acid (IAA) for $2 \mathrm{~h}$ prior to zeocin exposure to induce RPA degradation as shown in Supplemental Figure S2B. (D) Siz2 localizes to repair foci. Representative images for cells incubated with zeocin for $2 \mathrm{~h}$ are shown. The percentages of cells with $\mathrm{Rfa} 1$ or Siz2 foci and colocalizing foci before and after zeocin treatment are plotted. $(E)$ RPA is required for zeocin-induced Siz2 foci. Cells with or without TIR were incubated with $1 \mathrm{mM}$ IAA for $2 \mathrm{~h}$ prior to zeocin exposure. The percentages of cells with Siz2 foci were compared between cells containing Rfal (-TIR) and lacking Rfal (+TIR). (F) RPA depletion reduces Siz2 chromatin association after zeocin treatment. Cells were treated as in $C$, followed by chromatin fractionation. Pgk1 is a marker for soluble proteins, and Orc2 is a marker for chromatin-bound proteins. (W) Whole-cell extract; $(\mathrm{Su})$ supernatant; $(\mathrm{Ch})$ chromatin.

Rad52 and Rad59 was greatly reduced (Fig. 2C), while Siz2 levels or autosumoylation did not change (Supplemental Fig. S2C), suggesting that RPA loss reduces recombination protein sumoylation without affecting Siz2 enzymatic activity per se. This observation supports a direct or indirect role for RPA in Siz2-mediated sumoylation.

\section{$R P A$ is required for Siz2 repair foci formation}

Since human Siz2 homologs accumulate at DNA repair foci upon DSB generation (Galanty et al. 2009), we tested whether Siz2 also formed such foci and whether RPA might affect such localization. Live-cell imaging of yeast containing endogenous CFP- and YFP-tagged Rfa 1 and Siz2, respectively, confirmed that the percentage of cells containing nuclear Rfal foci increased more than twofold, from $17 \%$ to $38 \%$, upon zeocin treatment, as reported previously (Fig. 2D; Lisby and Rothstein 2009). An increase in cells with Siz2-YFP foci was also detected (from 3\% to 12\%). Importantly, almost all of the Siz2-YFP foci colocalized with Rfal foci before and after zeocin treatment (Fig. 2D). As $\mathrm{Rfal}$ foci are indicative of DNA repair centers (Lisby and Rothstein 2009), these findings show that Siz2, like its human homologs, is recruited to DNA lesion sites.
Using the above degron system, we found that RPA loss greatly reduced the number of cells containing Siz2 foci after zeocin treatment (from 10\% to 3\%) (Fig. 2E) without affecting Siz2 protein levels or sumoylation (Supplemental Fig. S2D). With a complementary approach, we found that Siz2 chromatin association also depended on RPA upon zeocin treatment. In the presence of RPA, $\sim 50 \%$ of the cellular Siz2 pool was bound to chromatin before and after treatment (-TIR) (Fig. 2F, Supplemental Fig. S2E); however, when RPA was degraded, the chromatinassociated pool of Siz2 was reduced after, but not before, zeocin treatment (+TIR) (Fig. 2F; Supplemental Fig. S2E). This finding, together with the foci results, indicates that RPA is required for Siz2 recruitment to damage sites. The fact that Siz2 chromatin association is affected by RPA loss only after DSB induction suggests that Siz2 is mobilized from nondamaged DNA sites and redistributed to lesion sites in an RPA-dependent manner.

\section{ssDNA generation is important for Siz2-mediated sumoylation and foci formation}

DNA damage signaling requires not only RPA but also ssDNA generated from lesion processing. In fact, RPAcoated ssDNA acts as a lesion-sensing platform for checkpoint kinases and ubiquitin enzymes (Zou and Elledge 2003; Davies et al. 2008). If RPA were indeed required for Siz2 recruitment to DSBs, one would predict that the ssDNA generated from DSB end processing would also be important. Thus, we asked whether the absence of DSB end resection proteins affected zeocin-induced sumoylation. In budding yeast, the Exo1 and Sgs1/Dna2 pathways act redundantly in long-range resection at DSBs (for review, see Symington 2014). We found that exo1 $\Delta$, but not $s g s 1 \Delta$, greatly reduced the sumoylation of Rfa1, Rad52, and Rad59, and concomitant deletion of EXO1 and SGS1 had no further effect (Supplemental Fig. S3A). As an Exo1 nuclease-dead (ND) mutant (D173A) (Moreau et al. 2001) also strongly impaired sumoylation (Fig. 3A), Exol promotes sumoylation via its nucleolytic activity. Consistent with this, deleting the DNA end-clipping enzyme Mre11 also reduced substrate sumoylation, while removing its partner Sae2 had a smaller effect (Supplemental Fig. S3B). These results show that ssDNA generation is important for zeocin-induced sumoylation and that the resection factors contribute to different levels. The strong effect of Exol nucleolytic activity may be due to the possibility that zeocin-generated nicks near DSBs enable Exol-mediated ssDNA generation independently of other resection factors.

As the loss of sumoylation of Rfa1, Rad52, and Rad59 in exo1 $\Delta$ and exo1-ND cells was greater than in siz2 $\Delta$ cells, we postulated that Exol-mediated ssDNA generation has multiple roles in zeocin-induced sumoylation, and one of these pertains to Siz2 function. We tested this idea by examining whether exo1s impairs Siz2 recruitment to DNA damage sites. exo1 $\Delta$ reduced the number of cells with Siz2 foci by $11 \%$ without affecting Siz2 protein or autosumoylation levels (Fig. 3B; Supplemental Fig. $\mathrm{S} 3 \mathrm{C})$. exo1 1 also reduced the number of cells with RPA foci by $10 \%$ after zeocin treatment (Fig. 3B). The extents of exo1 $1 \Delta$-mediated reduction in Siz2 and RPA foci were similar, supporting the idea that Exol-mediated ssDNA formation leads to RPA binding to ssDNA, which in turn recruits Siz2. Also consistent with this notion, the 

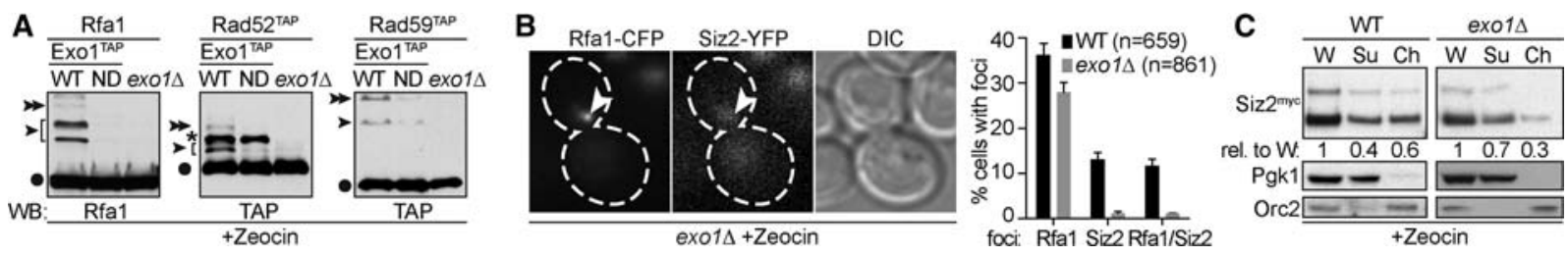

Figure 3. ssDNA generation is required for zeocin-induced sumoylation. $(A)$ Zeocin-induced sumoylation requires Exo1 and its nuclease activity. Note that the Exol ${ }^{\text {TAP }}$ band $(*)$ runs close to the monosumoylated form of Rad52. Experiments and presentation are as in Figure 1 A. $(B, C)$ exo1 reduces Rfa1 and Siz2 foci levels and Siz2 chromatin association after zeocin treatment. Experiments were done as in Figure 2, E andF.

proportion of chromatin-associated Siz2 in exo1 cells was reduced compared with wild type in the presence, but not absence, of zeocin (Fig. 3C; Supplemental Fig. S3D). Taken together, these data suggest that ssDNA generation and binding by RPA are required for Siz2 to localize to damaged DNA and sumoylate its substrates.

\section{Identification of Siz2 residues important for RPA interaction}

To further test the involvement of RPA in Siz2 function, we asked whether the RPA-Siz2 interaction per se was important for Siz2-mediated sumoylation and Siz2 lesion recruitment. Using Siz2 deletion constructs in $\mathrm{Y} 2 \mathrm{H}$, we mapped the Rfa2 interaction region to the first 120 residues encompassing the cSAP-eSAP region (Fig. 4A, \#14). As further deletion led to loss of interaction, the smaller fragments were likely folded incorrectly (data not shown). The first 120 residues of Siz2 and the Rfa2 C-terminal WH domain interacted in vitro in pull-down assays (Fig. 4B), demonstrating that the two domains can directly bind to each other. Consistent with the idea that this interaction is biologically important, conserved $\mathrm{Y} 2 \mathrm{H}$ interactions between the human Siz2 homologs PIAS1 and PIAS4 and the equivalent region of human Rfa2 (RPA2 172-270) were also detected (Fig. 4C).

Next, we determined the Siz2 residues important for $\mathrm{Rfa} 2$ interaction in several steps. First, we ruled out the possibility that the residues critical for DNA binding (mutated in cSAPmut) affect Rfa2 interaction using both $\mathrm{Y} 2 \mathrm{H}$ and co-IP assays (Supplemental Fig. S4A,B). Substantiating this conclusion, a chimeric construct with the cSAP-eSAP domain of Siz2 replaced with that of Siz1 $\left(\mathrm{SAP}_{\text {Siz1 }}\right)$ (Fig. 4A, \#5) failed to interact with Rfa2 despite retaining SUMO interaction. These results suggest that unique residues in the Siz2 cSAP-eSAP domain-but not those shared with Sizl, including the DNA-binding residues-are required for $\mathrm{Rfa} 2$ interaction.

Sequence alignment further revealed six amino acids in the cSAP-eSAP domain that are highly conserved among Siz2 orthologs but have properties very different from the corresponding residues in Sizl (Fig. 4D, blue boxes). Four residues are located in the eSAP region, while two are at the very $\mathrm{C}$ terminus of the cSAP region outside the DNA-interacting surface (Suzuki et al. 2009). To determine whether they support the Siz2-Rfa2 interaction, we mutated them to either alanine (6A: R72/R78/K92/ I103/K109/T119A) or residues different in charge or size (4E2K: R72E/R78E/K92E/I103K/K109E/T119K). Siz2-6A showed reduced $\mathrm{Rfa} 2$ interaction by $\mathrm{Y} 2 \mathrm{H}$, as cells grew on medium lacking histidine but not adenine (Fig. 4A, \#6), while Siz2-4E2K was more severely impaired for $\mathrm{Rfa} 2$ interaction (Fig. 4A, \#7). Consistent results were found by co-IP using chromosomally integrated versions of the mutants: Reduced amounts of Siz2-4E2K and Siz2-6A were recovered from Rfal immunoprecipitations compared with wild-type Siz2, with the 4E2K mutant showing a greater interaction defect (Fig. 4E,F; Supplemental Fig. S5A). We note that Siz2-4E2K protein and sumoylation levels were similar to those of wild type in these tests when using the GAL promoter (Supplemental Fig. S5B,C). These findings suggest that the six Siz2-specific residues are important for Siz2-RPA interaction. In conjunction with the finding that the DNA-binding mutations do not affect this interaction, it indicates that the cSAP-eSAP domain of Siz2 uses different residues for interacting with DNA and RPA.

\section{The Siz2-Rfa2 interaction is critical for Siz2 damage site recruitment and sumoylation}

Next, we examined whether the impaired Siz2-RPA interaction in the siz2-6A and siz2-4E2K mutants affected Siz2 function. Both mutants exhibited diminished zeocin-induced sumoylation of Rfa1, Rad52, and Rad59, with siz2-4E2K showing a stronger defect (Fig. 5A,B; Supplemental Fig. S6A), in accordance with its more severe loss of RPA interaction (Fig. 4A,F). Moreover, YFP-tagged Siz2-6A and Siz2-4E2K that were expressed at wild-type levels (Supplemental Fig. S6B) formed fewer foci compared with wild-type Siz2, whereas Rfa1-CFP foci formed at normal frequency (Fig. 5C; Supplemental Fig. S6C), indicating that RPA is still localized to DSBs, but Siz2 fails to be efficiently recruited in these mutant cells. Consistent with these findings, the Siz2 pool associated with chromatin was strongly reduced for the Siz2-6A and Siz2-4E2K proteins after zeocin treatment but not in untreated cells (Fig. 5D; Supplemental Fig. S6D). A similar observation was made with the Siz2-SAP ${ }_{\mathrm{Siz} 1}$ chimeric protein that failed to interact with RPA (Fig. 4A; Supplemental Fig. S6E). This further substantiates an RPA-mediated localization of Siz2 to chromatin specifically after DSB induction. In comparison, the Siz2-cSAPmut protein, which supports the Siz2-RPA interaction but is impaired for DNA binding (Supplemental Figs. S1C, S4A,B), exhibited wild-type levels of repair foci and chromatin association (Fig. 5C,D). Furthermore, the importance of the Siz2RPA interaction is reflected in the reduced viability of siz2-4E2K cells after zeocin treatment (Fig. 5E). Taken together, these results indicate that Siz2 interaction with RPA, but not DNA, is the main determinant for its recruitment to DNA break sites and its role in sumoylating recombination proteins.

Protein sumoylation provides an important means for the cell to up-regulate DNA repair and other recovery programs in the face of DNA stress situations (for review, see 
A

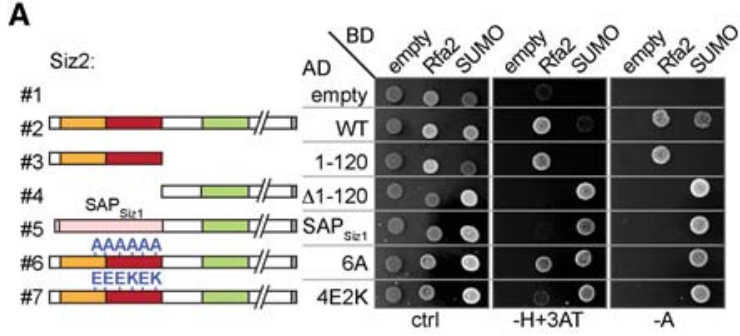

B

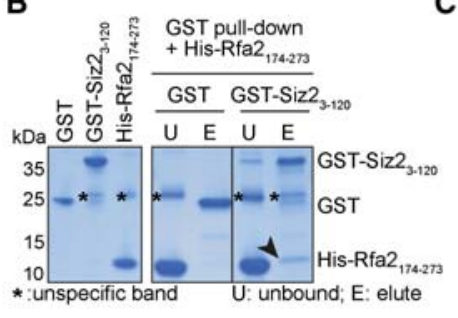

C

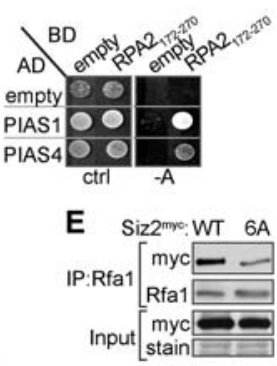

D S.cer 30: GGL INEI KDA INEMEQL KVLELKQICKSLDLSI TGKKAVLOLRI KQFLRKSCDIGHIDPNRFKA IKILIAKVFI ISSLPKYSTLWETLRTC S.par 27: GGL I NEI KDA INEMEQL KVLELKQI CKSLDLA I TGKKAVLQCRI KQFLRKSCDIGHIDPWRP KA I KIL IAKVKI NSSLPKYSTLWETLKT Z bai 12: RGLHOEI QEA IEQV DOL KVTELKSICRSIELPI TGRKAVLOCRIRVYL KNSCSIGHIDPWRPKT I RAL I DKVKL GEVLPKYDI LWOSLKS T.delb 11: RGLHHE I QEV NOMETLRVPEL KAVCRSLGQT IAGRKAELQER IRSWRNSCT IG WDPWRPKIVRLLL I DKVKL GETLPSYEDWMTLPTC

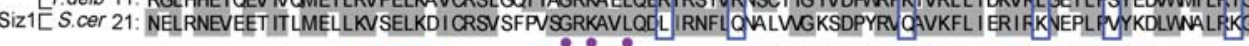
00 - DNA interaction residues -mutated in Siz2-cSAPmut

Figure 4. Mapping of the Siz2-RPA interaction residues. (A) Siz2 mutants examined for Rfa2 interaction by Y2H. Binding to SUMO was used as a control. Growth on $-\mathrm{H}+3 \mathrm{AT}$ and $-\mathrm{A}$ plates indicates differences in interaction strength. (B) The Siz2 cSAP-eSAP domain (amino acids 3-120) and the Rfa2 C terminus (amino acids 174-273) bind in vitro. (C) Human Siz2 homologs PIAS1 and PIAS4 interact with the C-terminal fragment of human RPA2 by Y2H. (D) Alignment of residues 30-120 of Saccharomyces cerevisiae (S. cer) Siz2 with similar regions of its close orthologs and S. cer Siz1. Identical or similar residues are shaded in gray. The predicted Siz2 secondary structure is shown at the top. Siz1 residues contacting DNA (orange circles) (Suzuki et al. 2009) or abolishing DNA binding in cSAPmut (violet circles) are indicated. Conserved residues of Siz2 orthologs that are different in Sizl were mutated in siz2-6A and siz2-4E2K (blue boxes). (S. par) Saccharomyces paradoxus; (Z. bai) Zygosaccharomyces bailii; (T. del) Torulaspora delbrueckii. $(E, F)$ siz2-6A and siz2-4E2K reduce RPA interaction. RPA was pulled down with an anti-Rfa 1-antibody (control immunoprecipitation in Supplemental Fig. S5A), and coimmunoprecipitated Siz2 was detected via its myc tag. In $F$, the GAL promoter was used to ensure the same protein levels between siz2-4E2K and wild type (see Supplemental Fig. S5B).

Sarangi and Zhao 2015). One of the very first steps in this response is the localization of sumoylation enzymes to DNA lesion sites. Here, we provide important insight into the mechanism of this crucial process. Our findings suggest that the SUMO-mediated DDR is enabled by collaboration between a SUMO ligase and the DNA damagesensing complex RPA. We show that the SUMO ligase Siz2 is recruited to repair foci upon DSB generation. This localization and Siz2-mediated sumoylation require both ssDNA-generating enzymes and the ssDNA-binding complex RPA. We uncovered a conserved interaction between Siz2 and RPA that is mediated by direct binding between the cSAP-eSAP domain of Siz2 and the middle subunit of RPA. Mutating key residues responsible for this interaction diminished Siz2 repair foci formation, chromatin association, substrate sumoylation, and cell survival after DSB induction. These findings suggest that ssDNA-coated RPA serves as a recruitment platform for the Siz2 SUMO ligase to enable it to modify its substrates at lesion sites (Fig. 5F).

The presence of large amounts of ssDNA correlates with genome stress; thus, RPA-coated ssDNA is perfectly suited for initiating the damage-sensing process and recruiting PTM enzymes. Indeed, RPA interacts with and recruits the checkpoint kinase complex Mec1-Ddc2 (human ATRATRIP) and the ubiquitin ligase Rad18 (Zou and Elledge 2003; Davies et al. 2008). Our discovery of a similar mechanism for a SUMO ligase unifies the theme of PTM enzyme recruitment in DDR and suggests that RPA-coated ssDNA triggers three types of PTMs: phosphorylation, ubiquitination, and sumoylation. While our findings pertain to zeocin treatment, the collaboration of RPA and SUMO enzymes in inducing sumoylation is likely a general principle. In support of this idea, we found that the siz2$4 E 2 K$ mutant impaired for RPA binding was defective in inducing sumoylation of Rfa1, Rad52, and Rad59 under MMS treatment as well (Supplemental Fig. S6F). As human PIAS1/4 proteins also interact with the RPA middle subunit (Fig. 4C) and contain a homologous cSAP-eSAP domain, PIAS proteins likely use an analogous mechanism to induce sumoylation upon DNA damage.

Unlike most other PTM enzymes that bind to the large subunit of RPA, the Siz2 SUMO ligase interacts with the middle subunit of RPA, suggesting that the trimeric RPA complex could use different surfaces to concurrently recruit different types of PTM enzymes. As RPA subunits also interact with DNA repair factors (Marechal and Zou 2015), the RPA nucleofilament can synergize the functions of PTM enzymes and repair proteins by bringing them in close proximity. Although the DNA-binding feature of cSAP is not required for Siz2 function upon zeocin treatment, it may enhance RPA-mediated enzyme recruitment under other conditions. Alternatively, since mutations in the DNA-binding residues of both Siz1 and Siz2 result in lower protein levels (Supplemental Fig. S1B; Parker et al. 2008; Psakhye and Jentsch 2012), DNA interaction might generally promote their stability. As only Siz2 mediates DNA break-induced sumoylation of the tested substrates even though Sizl also interacts with DNA, specific protein-protein interactions dictate ligase localization and substrate specificity.

In summary, the revelation of RPA-ssDNA as a landing platform for Siz2 provides a molecular basis for the recruitment of SUMO ligases to DNA damage sites. This finding unifies a general paradigm of PTM enzyme recruitment in DDR. Considering the highly conserved protein structures and functions among the PIAS ligases, it is likely that the mechanisms discovered here are conserved throughout evolution.

\section{Materials and methods}

The strains used here are listed in Supplemental Table S1. Standard protocols were used for yeast work. Zeocin $(0.3 \mathrm{mg} / \mathrm{mL}$; Life Technologies) was added to log-phase cultures for $2 \mathrm{~h}$ before cells were collected. For the induction of GAL-siz2-cSAPmut and GAL-siz2-4E2K, cells were grown in YP-raffinose, and galactose was added for $3 \mathrm{~h}$. Degradation of Rfal-AID was induced with $1 \mathrm{mM}$ IAA (Sigma) for $2 \mathrm{~h}$. Y2H assays were performed as 


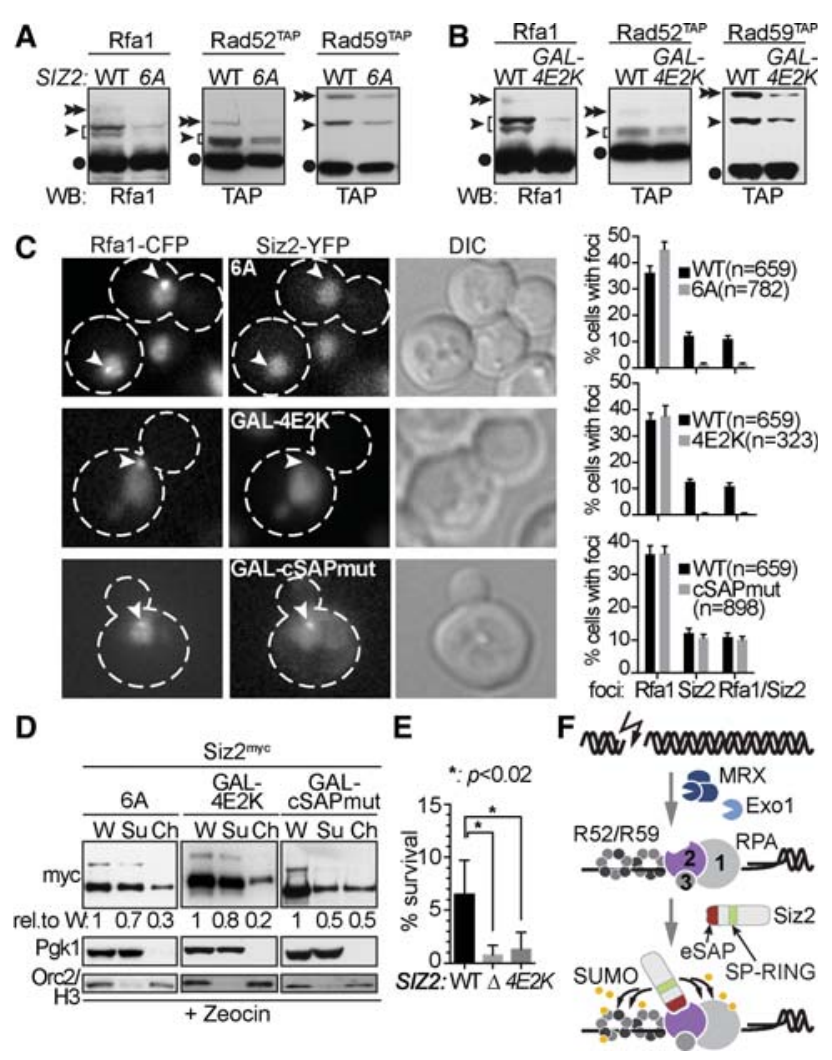

Figure 5. Siz2-RPA interaction promotes Siz2-mediated sumoylation, Siz2 foci formation, chromatin association, and cellular survival after zeocin treatment. $(A, B)$ Zeocin-induced sumoylation of Rfal, Rad52, and Rad59 is decreased in the siz2-6A and siz2-4E2K mutants. For quantification, see Supplemental Figure S6A. (C) Percentages of cells with Rfa1 and Siz2 foci after zeocin treatment in mutants of the indicated Siz2-YFP variants (as in Fig. 2D, except representative images of wild-type cells are in Supplemental Fig. S6C). (D) Siz2 mutants defective in RPA interaction, but not DNA binding, show reduced chromatin association. Fractionation was carried out as in Figure 2F. (E) siz2-4E2K and siz2A cells show reduced viability after zeocin treatment. $(F)$ A model for SUMO ligase recruitment to DNA lesions. DSB resection by enzymes such as the Mre11 complex (MRX) and Exol generates ssDNA. RPA-coated ssDNA recruits SUMO ligases via an interaction between the eSAP domain of the enzyme (red) and the middle subunit of RPA (purple). Close proximity allows Siz2 to sumoylate repair proteins via its SP-RING domain (green).

described previously (Sarangi et al. 2014). The strength of interaction was determined by cell growth on $-\mathrm{His}+3 \mathrm{AT}$ (or $-\mathrm{H}+3 \mathrm{AT}$ ) and $-\mathrm{Ade}$ (or $-\mathrm{A}$ ) plates. Several techniques were done as previously described and are detailed in the Supplemental Material: protein preparation and detection (Sarangi et al. 2014), chromatin fractionation (Liang and Stillman 1997), and live-cell imaging (Chen et al. 2009). Protein purification, GST pull-down, and gel shift assays followed standard procedures and are described in the Supplemental Material. For accurate quantification of protein band intensities, Western blots were scanned using an LAS-3000 Imager (Fujifilm), which has a dynamic range of four orders of magnitude, and the signal intensities of nonsaturated bands were measured using ImageGauge software.

\section{Acknowledgments}

We thank Yoona Lee, Lei Wei, Yihu Xie, and Soon Bahng for technical assistance; Prabha Sarangi and Catherine Cremona for helpful comments; and L. Symington and H. Zhou for plasmids. We are grateful to S. Brill for sharing anti-Rfal antibody. We apologize for not citing the work of some colleagues due to space limitations. This work was supported by American Cancer Society grant RSG-12-013-01-CCG, National Institutes of Health grant GM080670, and a Leukemia and Lymphoma Society Scholar Award to X.Z., and the German Research Foundation (Deutsche Forschungsgemeinschaft; CH 1243/1-1) to I.C.

\section{References}

Brill SJ, Stillman B. 1991. Replication factor-A from Saccharomyces cerevisiae is encoded by three essential genes coordinately expressed at $\mathrm{S}$ phase. Genes Dev 5: 1589-1600.

Chen YH, Choi K, Szakal B, Arenz J, Duan X, Ye H, Branzei D, Zhao X. 2009. Interplay between the Smc5/6 complex and the Mph1 helicase in recombinational repair. Proc Natl Acad Sci 106: 21252-21257.

Davies AA, Huttner D, Daigaku Y, Chen S, Ulrich HD. 2008. Activation of ubiquitin-dependent DNA damage bypass is mediated by replication protein A. Mol Cell 29: 625-636.

Galanty Y, Belotserkovskaya R, Coates J, Polo S, Miller KM, Jackson SP. 2009. Mammalian SUMO E3-ligases PIAS1 and PIAS4 promote responses to DNA double-strand breaks. Nature 462: 935-939.

Johnson ES, Gupta AA. 2001. An E3-like factor that promotes SUMO conjugation to the yeast septins. Cell 106: 735-744.

Liang C, Stillman B. 1997. Persistent initiation of DNA replication and chromatin-bound MCM proteins during the cell cycle in cdc6 mutants. Genes Dev 11: 3375-3386.

Lisby M, Rothstein R. 2009. Choreography of recombination proteins during the DNA damage response. DNA Repair 8: 1068-1076.

Marechal A, Zou L. 2015. RPA-coated single-stranded DNA as a platform for post-translational modifications in the DNA damage response. Cell Res 25: 9-23.

Moreau S, Morgan EA, Symington LS. 2001. Overlapping functions of the Saccharomyces cerevisiae Mre11, Exo1 and Rad27 nucleases in DNA metabolism. Genetics 159: 1423-1433.

Morris JR, Boutell C, Keppler M, Densham R, Weekes D, Alamshah A, Butler L, Galanty Y, Pangon L, Kiuchi T, et al. 2009. The SUMO modification pathway is involved in the BRCA1 response to genotoxic stress. Nature 462: 886-890.

Nishimura K, Fukagawa T, Takisawa H, Kakimoto T, Kanemaki M. 2009. An auxin-based degron system for the rapid depletion of proteins in nonplant cells. Nat Methods 6: 917-922.

Okubo S, Hara F, Tsuchida Y, Shimotakahara S, Suzuki S, Hatanaka H, Yokoyama S, Tanaka H, Yasuda H, Shindo H. 2004. NMR structure of the N-terminal domain of SUMO ligase PIAS1 and its interaction with tumor suppressor p53 and A/T-rich DNA oligomers. I Biol Chem 279: 31455-31461.

Parker JL, Bucceri A, Davies AA, Heidrich K, Windecker H, Ulrich HD. 2008. SUMO modification of PCNA is controlled by DNA. EMBO $I$ 27: 2422-2431.

Psakhye I, Jentsch S. 2012. Protein group modification and synergy in the SUMO pathway as exemplified in DNA repair. Cell 151: 807-820.

Rytinki MM, Kaikkonen S, Pehkonen P, Jaaskelainen T, Palvimo JJ. 2009. PIAS proteins: pleiotropic interactors associated with SUMO. Cell Mol Life Sci 66: 3029-3041.

Sarangi P, Zhao X. 2015. SUMO-mediated regulation of DNA damage repair and responses. Trends Biochem Sci 40: 233-242.

Sarangi P, Altmannova V, Holland C, Bartosova Z, Hao F, Anrather D, Ammerer G, Lee SE, Krejci L, Zhao X. 2014. A versatile scaffold contributes to damage survival via sumoylation and nuclease interactions. Cell Rep 9: 143-152.

Suzuki R, Shindo H, Tase A, Kikuchi Y, Shimizu M, Yamazaki T. 2009. Solution structures and DNA binding properties of the $\mathrm{N}$-terminal SAP domains of SUMO E3 ligases from Saccharomyces cerevisiae and Oryza sativa. Proteins 75: 336-347.

Symington LS. 2014. End resection at double-strand breaks: mechanism and regulation. Cold Spring Harb Perspect Biol 6: a016436.

Zou L, Elledge SJ. 2003. Sensing DNA damage through ATRIP recognition of RPA-ssDNA complexes. Science 300: 1542-1548. 


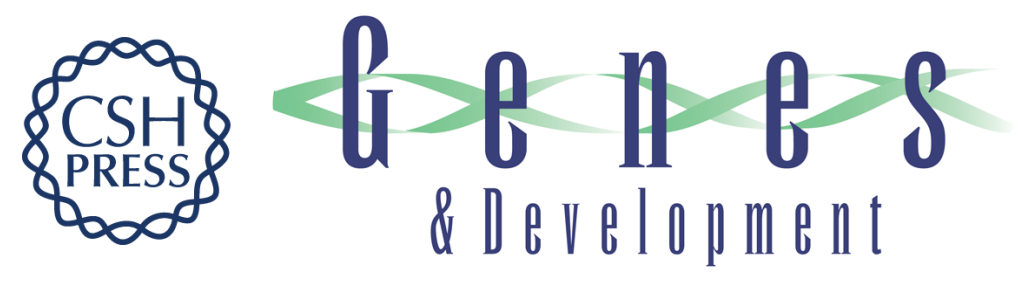

\section{DNA break-induced sumoylation is enabled by collaboration between a SUMO ligase and the ssDNA-binding complex RPA}

Inn Chung and Xiaolan Zhao

Genes Dev. 2015, 29:

Access the most recent version at doi:10.1101/gad.265058.115

\section{Supplemental http://genesdev.cshlp.org/content/suppl/2015/08/07/29.15.1593.DC1 \\ Material}

References This article cites 20 articles, 8 of which can be accessed free at:

http://genesdev.cshlp.org/content/29/15/1593.full.html\#ref-list-1

Creative This article is distributed exclusively by Cold Spring Harbor Laboratory Press for the first

Commons six months after the full-issue publication date (see

License http://genesdev.cshlp.org/site/misc/terms.xhtml). After six months, it is available under a Creative Commons License (Attribution-NonCommercial 4.0 International), as described at http://creativecommons.org/licenses/by-nc/4.0/.

Email Alerting Receive free email alerts when new articles cite this article - sign up in the box at the top Service right corner of the article or click here.

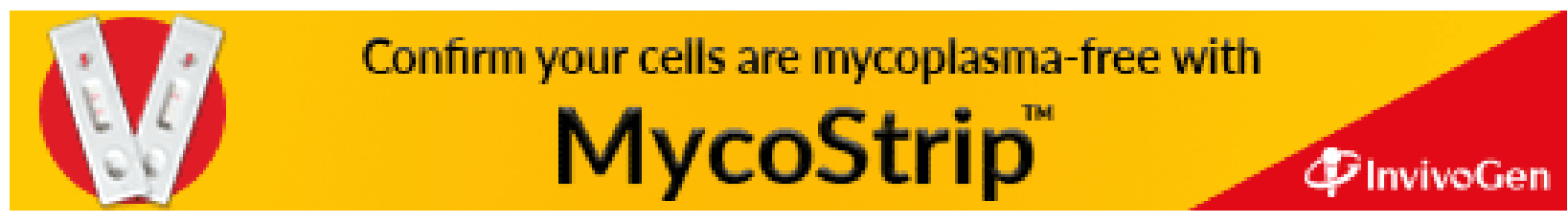

